\title{
RESEARCH
}

Open Access

\section{FGF21 alleviates neuroinflammation following ischemic stroke by modulating the temporal and spatial dynamics of microglia/macrophages}

Wang Dongxue ${ }^{1,2}$, Liu Fei ${ }^{2}$, Zhu Liyun ${ }^{2}$, Lin Ping ${ }^{2}$, Han Fanyi ${ }^{2}$, Wang Xue ${ }^{2}$, Tan Xianxi ${ }^{1}$, Lin Li $i^{1,2^{*}}$ and Xiong Ye ${ }^{1 *}$ (D)

\begin{abstract}
Background: Resident microglia and macrophages are the predominant contributors to neuroinflammation and immune reactions, which play a critical role in the pathogenesis of ischemic brain injury. Controlling inflammatory responses is considered a promising therapeutic approach for stroke. Recombinant human fibroblast growth factor 21 (rhFGF21) presents anti-inflammatory properties by modulating microglia and macrophages; however, our knowledge of the inflammatory modulation of rhFGF21 in focal cerebral ischemia is lacking. Therefore, we investigated whether rhFGF21 improves ischemic outcomes in experimental stroke by targeting microglia and macrophages.

Methods: C57BL/6 mice were subjected to middle cerebral artery occlusion (MCAO) and randomly divided into groups that received intraperitoneal rhFGF21 or vehicle daily starting at $6 \mathrm{~h}$ after reperfusion. Behavior assessments were monitored for 14 days after MCAO, and the gene expression levels of inflammatory cytokines were analyzed via qRT-PCR. The phenotypic variation of microglia/macrophages and the presence of infiltrated immune cells were examined by flow cytometry and immunostaining. Additionally, magnetic cell sorting (MACS) in combination with fluorescence-activated cell sorting (FACS) was used to purify microglia and macrophages.
\end{abstract}

Results: rhFGF21 administration ameliorated neurological deficits in behavioral tests by regulating the secretion of proinflammatory and anti-inflammatory cytokines. rhFGF21 also attenuated the polarization of microglia/macrophages toward the M1 phenotype and the accumulation of peripheral immune cells after stroke, accompanied by a temporal evolution of the phenotype of microglia/macrophages and infiltration of peripheral immune cells. Furthermore, rhFGF21 treatment inhibited M1 polarization of microglia and pro-inflammatory cytokine expression through its actions on FGF receptor 1 (FGFR1) by suppressing nuclear factor-kappa B (NF-KB) and upregulating peroxisome proliferatoractivated receptor- $\gamma$ (PPAR- $\gamma$ ).

Conclusions: rhFGF21 treatment promoted functional recovery in experimental stroke by modulating microglia/ macrophage-mediated neuroinflammation via the NF-KB and PPAR- $\gamma$ signaling pathways, making it a potential antiinflammatory agent for stroke treatment.

Keywords: rhFGF21, Stroke, Neuroinflammation, Microglia/macrophage, NF-kB, PPAR- $\gamma$

\footnotetext{
*Correspondence: linliwz@163.com; xiongye2310@163.com

'Department of Neurosurgery, First Affiliated Hospital of Wenzhou Medical University, Wenzhou 325035, Zhejiang, China

Full list of author information is available at the end of the article
}

C C The Author(s). 2020 Open Access This article is licensed under a Creative Commons Attribution 4.0 International License, which permits use, sharing, adaptation, distribution and reproduction in any medium or format, as long as you give appropriate credit to the original author(s) and the source, provide a link to the Creative Commons licence, and indicate if changes were made. The images or other third party material in this article are included in the article's Creative Commons licence, unless indicated otherwise in a credit line to the material. If material is not included in the article's Creative Commons licence and your intended use is not permitted by statutory regulation or exceeds the permitted use, you will need to obtain permission directly from the copyright holder. To view a copy of this licence, visit http://creativecommons.org/licenses/by/4.0/ The Creative Commons Public Domain Dedication waiver (http://creativecommons.org/publicdomain/zero/1.0/) applies to the data made available in this article, unless otherwise stated in a credit line to the data. 


\section{Introduction}

Ischemic stroke, reduced cerebral blood flow caused by an arterial thrombus, which afflicts approximately 795 , 000 individuals worldwide each year, and the number of patients suffering stroke is rising [1, 2]. However, the therapeutic options for stroke are desperately limited. Slow and incomplete recovery is compounded by limited drug treatments that facilitate the recovery process. Acute care mainly depends on thrombolytic treatment by administrating tissue plasminogen activator (tPA), although the narrow therapeutic time window of within 6 $\mathrm{h}$ ensures that only a small fraction of patients benefit [3]. Furthermore, reperfusion of the ischemic brain is considered a secondary injury, and the efficacy of tPA treatment is inconsistent among individuals. Consequently, novel and effective drug treatments that improve the symptoms and sequelae of stroke, especially in acute phases, are urgently needed [4].

Inflammation is a critical component of the secondary injury resolution process under ischemic brain insult. In the event of stroke, microglia residing in the central nervous system (CNS) are the first responders to cerebral ischemia, and they are activated within several minutes $[5,6]$. Subsequently, infiltrating immune cells, including monocytes, macrophages, neutrophils, lymphocytes, and natural killer (NK) cells, pass through the disrupted blood-brain barrier (BBB) and secrete a plethora of cytokines to promote the progression of inflammation $[7,8]$. Therefore, understanding the contribution of those immune cells to the immunomodulation reaction is a prerequisite for therapeutic intervention. In particular, resident microglia, as well as invading macrophages, are commonly recognized as vital contributors to inflammatory circumstances under the pathophysiology of ischemic stroke [9]. Morphological transformation and common antigens expressed on both cell types give them certain overlapping functions, such as phagocytosis and analogous polarization abilities toward M1- or M2-like phenotypes $[6,10]$. The diverse phenotypes distinctively impact the expression of inflammatory cytokines, which are correlated with neuronal functions. Generally, the M1 microglia/macrophages, marked CD16/32 and CD68, are commonly characterized by pro-inflammatory effects accompanied by the release of pro-inflammatory cytokines including tumor necrosis factor- $\alpha$ (TNF- $\alpha$ ), inducible nitric oxide synthase (iNOS), interleukin-1 $\beta$ (IL-1 $\beta$ ), and interleukin-6 (IL-6), whereas microglia with the M2 phenotype (marked by CD206) secrete transforming growth factor beta (TGF- $\beta$ ), insulin-like growth factor 1 (IGF-1), interleukin (IL)-10, and IL-4 to rescue local inflammation and favor tissue repair $[11,12]$. Furthermore, the M2 phenotype is divided into three subsets: M2a (marked by CD206 and arginase-1) is associated with anti-inflammation and immunity against parasites, M2b (marked by CD86 and SOCS3) is related to adaptive immunity, and M2c (marked by TGF- $\beta$ and IL-10) facilitates tissue regeneration [13]. Notably, the unique temporal and spatial changes in microglia and macrophages under pathophysiological conditions indicate that each cell type has indispensable and complementary roles in the context of ischemic stroke. A growing number of studies have focused on the phenotypic moderation of microglia/ macrophages rather than the exclusive suppression of their activation. However, the participation of invading immune cells is usually obscured.

Fibroblast growth factor 21 (FGF21), as a novel and potent regulator of glucose uptake and lipid metabolism, is predominantly expressed in both the rodent and human liver and thymus [14]. Compared with other FGFs, FGF21 scarcely has mitogenic effects and may be the only FGF that can cross the BBB due to its weak binding affinity with heparin $[15,16]$. To date, accumulating evidence has indicated that FGF21 exhibited therapeutic effects in multiple disease models, such as atherosclerosis [17], diabetic cardiomyopathy [18], agerelated disorders [19], and enhanced neurite outgrowth [20]. Although the mechanisms underlying its pharmacologic actions remain elusive, the therapeutic mechanism of FGF21 primarily involves anti-inflammation [21], energy metabolism and vascular homeostasis [22], oxidative stress [23], and tissue repair [24]. FGF21 mediates these effects by interacting with FGF receptors (mainly FGFR1 and FGFR2) via binding a cofactor, $\beta$-klotho, a single-pass transmembrane protein from the klotho family [25]. FGFR1 and its coreceptor $\beta$-klotho have also been reported to be widely observed in brain tissue, including microglia [26]. Therefore, FGF21 may have a potential impact on microglia. Additionally, our previous study demonstrated that FGF21 effectively upregulated the downstream effector peroxisome proliferator-activated receptor (PPAR) $-\gamma$ in human bone marrow endothelial cells [27] and activated PPAR- $\gamma$ was closely associated with microglial phenotype and inflammatory regulation in the CNS [28]. Moreover, a recent study confirmed that FGF21 suppressed macrophage-mediated inflammation by nuclear factor-erythroid 2-related factor 2 (Nrf2) and the NF- $\mathrm{kB}$ signaling pathway in a collagen-induced arthritis model [29].

Therefore, FGF21 is likely to regulate the strokeinduced immune-inflammatory response by modulating microglia and macrophages both in the brain and in peripheral tissue in favor of functional recovery. Recently, recombinant human FGF21 (rhFGF21) has been reported to modulate the shift of microglia from M1 activation to M2 activation at the subacute and chronic stages of $\mathrm{db} / \mathrm{db}$ mice with middle cerebral artery occlusion (MCAO), which is accompanied by the activation of PPAR- $\gamma$ in the peri-infarct area [30]. However, the 
potential mechanism by which FGF21 acts on microglia/ macrophages and the dynamic alteration of microglia/ macrophages and their phenotypes have not been elucidated. In the current study, we investigated the neuroprotective effect and promising mechanism by which FGF21 ameliorates inflammatory responses and microglia/macrophage polarization in a mouse model of MCAO.

\section{Materials and methods}

Reagents and antibodies

rhFGF21 was supported by the laboratory of Biotechnology Pharmaceutical Engineering at Wenzhou Medical University and synthesized on the basis of the study previously reported [31]. Antibodies in flow cytometry analysis involving CD3-PE (17A2, 100206), CD3-PerCPCy5.5 (17A2, 100217), CD8-FITC (53-5.8, 140404), CD4-APC (GK1.5, 100412), F4/80-FITC (BM8, 123108), NK1.1-APC (PK136, 180710), Ly6G-PE (1A8, 127608), Ly6C-APC (HK1.4, 128016), CD45-APC (103112), CD11b-PE (101208), CD206-APC (C068C2, 141708), CD206-FITC (C068C2, 141704), CD68-PerCP-Cy5.5 (FA-11, 137014), CD86-PE (GL-1, 105008), CD45-PE/ Cy7 (30-F11, 103114), CD11b-PE/Cy7 (M1/70, 101216), and CD11b-PerCP-Cy5.5 (M1/70, 101230) were purchased from BD Biosciences (San Jose, CA, USA) and CD45-APC (OX33, 17046280), CD11b-PE (OX42, 12011080), and CD86-FITC (24F, 11086081) were purchased from eBioscience (San Diego, CA, USA).

Antibodies applied in immunofluorescence including CD16/32(AF1460) and CD206 (AF2535) were purchased from R\&D Systems (Minneapolis, MN, USA) and Iba1 (019-19741) purchased from Wako pure chemical corporation (Tokyo, Japan).

The primary antibodies applied in western blot including anti-NF-kB (3033T), anti-FGFR1 (ab824), anti-p-FGFR1 (ab59194), anti-PPAR- $\gamma$ (ab28364), and anti- $\beta$-Actin (ab8227) were purchased from Cell Signaling Technology (Danvers, MA, USA) or Abcam (Cambridge, MA, USA). The secondary antibodies used were donkey anti-rabbit IgG H\&L (HRP) (ab150075) or goat anti-mouse IgG H\&L (HRP) (ab150115), which were commercially purchased from Abcam (Cambridge, MA, USA).

Corresponding reagent or kit applied in this study include trizol reagent (Qiagen, Duesseldorf, Germany), PrimeScript $^{\mathrm{TM}}$ RT Reagent Kit (TaKaRa, Shiga, Japan), $\mathrm{iQ}^{\mathrm{TM}}$ SYBR Green supermix (Bio-Rad, Hercules, CA, USA), miRNeasy Micro Kit (Qiagen, Duesseldorf, Germany), QuantiTect Reverse Transcription kit (Qiagen, Duesseldorf, Germany), TaqMan ${ }^{\circ}$ Gene Expression Assays (ThermoFisher Scientific, Fremont, CA, USA), Neural Tissue Dissociation Kits (Miltenyi Biotech, Bergisch Gladbach Germany), and Fluoroshield mounting medium with DIPI (Abcam, Cambridge, MA, USA).

\section{Animal groups and drug administration}

C57BL/6 mice (20-25 g) were purchased from the Animal Center of the Chinese Academy of Science (Beijing, China), and all surgical procedures and experimental protocols were approved by the Animal Care and Use Committee of Wenzhou Medical University. All animals were randomly assigned to the following three groups by a randomized block design: sham group, MCAO group, and MCAO+ rhFGF21 group. In the sham group, mice were subjected to the same anesthesia and surgical procedures as the other groups but the filament was not inserted. In the MCAO+ rhFGF21 group, the mice were intraperitoneally injected with rhFGF21 once per day at a dose of $1.5 \mathrm{mg} / \mathrm{kg}$ for 7 consecutive days beginning at $6 \mathrm{~h}$ after reperfusion.

\section{Transient focal cerebral ischemia and reperfusion model preparation}

The surgical procedures to establish the MCAO model were based on the intraluminal filament technique [32]. Briefly, the mice were anesthetized by isoflurane and placed on a heating blanket to maintain body temperature at $37 \pm 0.5^{\circ} \mathrm{C}$. A midline incision was made to expose the common carotid artery (CCA), external carotid artery (ECA), and internal carotid artery (ICA). The CCA was temporarily closed and a monofilament $(0.18 \pm 0.01 \mathrm{~mm}$, Jialing Biotechnology Company, Guangdong, China) was inserted into the ICA through the ECA until it reached the middle cerebral artery, and it was left for $60 \mathrm{~min}$. Laser Doppler flowmetry (model P10, Moor Instruments, Wilmington, DE, USA) was used to monitor whether cerebral flow dropped to lower than $20 \%$ of the pre-ischemic level. The occluding filament was returned to the ICA to achieve reperfusion after $60 \mathrm{~min}$ of occlusion. In the MCAO model, the mortality rate was $9.3 \%$ (23 of total 246) and exclusion rate was $10.9 \%$ (11 of the total 246 experienced inadequate reperfusion, and 16 of the total 246 reached the criteria limitations set for the modified Neurological Severity Score (mNSS) scoring system, i.e., $\mathrm{mNSS}$ scores $<6$ or $>$ 13 at $24 \mathrm{~h}$ after MCAO were excluded).

\section{Neurological function assessment}

The mNSS, rotarod test, corner-turning test, and adhesive removal test were performed to assess neurodeficits, motor coordination, sensorimotor asymmetry, and feeling functions at $1,3,7$, and 14 days after surgery. All animals received training for 3 consecutive days before suffering ischemia-reperfusion injury. Behavior data were recorded as preoperative data at the second day after training. Subsequently, a transient focal cerebral ischemia and reperfusion model about MCAO was performed on the following day. The assessment procedure was performed by the same investigator who was blinded to the group identity of each mouse. 


\section{Quantitative real-time PCR}

Total mRNA was isolated from the cortex samples around the infarcted zone using trizol reagent according to the manufacturer's instructions. The cDNA was synthesized by the PrimeScript ${ }^{\mathrm{TM}}$ RT Reagent Kit (TaKaRa, Shiga, Japan) following the manufacturer's protocol. PCR assays were performed on a CFX Connect Real-time System (Bio-Rad, Hercules, CA, USA) using SYBR Green. The primers used in this study are shown in Table 1. Additionally, total RNA was extracted from the sorted microglia using the miRNeasy Micro Kit according to the manufacturer's protocol. cDNA was transcribed with a Reverse Transcription kit and amplified in step one using Gene Expression Assays for TNF- $\alpha$, IL-6, IL-1 $\beta$, and TGF- $\beta$. The reaction volume was set to $20 \mu \mathrm{l}$ and performed at $50^{\circ} \mathrm{C}$ for $2 \mathrm{~min}, 95^{\circ} \mathrm{C}$ for $20 \mathrm{~s}$, followed by 40 cycles of $1 \mathrm{~s}$ at $95^{\circ} \mathrm{C}$ and $20 \mathrm{~s}$ at $60^{\circ} \mathrm{C}$. Data were analyzed using the $2^{-\Delta \Delta \mathrm{Ct}}$ method, and the expression level of relative mRNA was then reported as the fold difference.

\section{Flow cytometry}

After the mice were euthanized, fresh brain, spleen, and blood tissues were harvested for single-cell suspension preparation for subsequent single-cell analysis using fluorochrome-conjugated antibodies. Spleen and blood tissues were dissociated into single-cell suspensions as previously described [33, 34]. Splenocytes were dissociated by sieving through a $70-\mu \mathrm{m}$ filter, and then lysing solution (BD Bioscience, CA, USA) was used to deplete red blood cells in the spleen and blood. Brain mononuclear cells were prepared by Neural Tissue Dissociation Kits (Miltenyi Biotech,

Table 1 PPrimers sequences for qRT-PCR

\begin{tabular}{|c|c|}
\hline Gene & Primer sequences ( $5^{\prime}$ to $\left.3^{\prime}\right)$ \\
\hline \multirow[t]{2}{*}{$\beta$-Actin } & Forward CACTGCAAACGGGGAAATGG \\
\hline & Reverse TGAGATGGACTGTCGGATGG \\
\hline $\mathrm{IL}-1 \beta$ & $\begin{array}{l}\text { Forward GCG CTG CTC AAC TTC ATC TTG } \\
\text { Reverse GTG ACA CAT TAA GCG GCT TCA C }\end{array}$ \\
\hline \multirow[t]{2}{*}{ IL-6 } & Forward CTC CCA ACA GAC CTG TCT ATA C \\
\hline & Reverse CCA TTG CAC AAC TCT TाT СTC A \\
\hline \multirow[t]{2}{*}{ TNF-a } & Forward GTG ACA AGC CTG TAG CCC A \\
\hline & Reverse ACT CGG CAA AGT CGA GAT AG \\
\hline \multirow[t]{2}{*}{ Cox-2 } & Forward CCCTTGGGTGTCAAAGGTAA \\
\hline & Reverse GCCCTCGCTTATGATCTGTC \\
\hline MCP-1 & $\begin{array}{l}\text { Forward ATAGCAGCCACCTTCATTCC } \\
\text { Reverse TTCCCCAAGTCTCTGTATCT }\end{array}$ \\
\hline CXCL1 & $\begin{array}{l}\text { Forward ACC GAA GTC ATA GCC ACA CCTC AAG } \\
\text { Reverse TTG TCA GAA GCC AGC GTT CAC C }\end{array}$ \\
\hline \multirow[t]{2}{*}{$\mid \mathrm{L}-10$} & Forward TTC TाT CAA ACA AAG GAC CAG C \\
\hline & Reverse GCA ACC CAA GTA ACC CTT AAA G \\
\hline \multirow[t]{2}{*}{ TGF- $\beta$} & Forward TTGCTTGAGCTCCACAGAGA \\
\hline & Reverse TGGTTGTAGAGGGCAAGGAC \\
\hline
\end{tabular}

Bergisch Gladbach Germany) according to its protocol. Briefly, the ischemic hemisphere of the brain was collected and dissected into small pieces. The pieces were pipetted back into an appropriate-sized conical tube, rinsed with cold Hank's balanced salt solution (HBSS), and then centrifuged ( $300 \mathrm{~g}, 2 \mathrm{~min})$ at room temperature. After the supernatant was carefully aspirated, preheated enzyme mix1 $\left(37^{\circ} \mathrm{C}, 10 \mathrm{~min}\right)$ in a Neural Tissue Dissociation Kit was added to digest tissue pieces for $15 \mathrm{~min}$, and then preheated enzyme mix $2\left(37^{\circ} \mathrm{C}, 10 \mathrm{~min}\right)$ was added to the tissue sample for $10 \mathrm{~min}$. Subsequently, HBSS was used and single pellets were isolated by passing through a $30-\mu \mathrm{m}$ cell strainer. Cell pellets obtained from the spleen, blood, and brain were washed and incubated with antibodies targeting CD3, CD8, CD4, F4/80, NK1.1, Ly6G, Ly6C, CD45, CD11b, CD206, CD68, and CD86, and tagged with phycoerythrin (PE), fluorescein isothiocyanate (FTIC), allophycocyanin (APC), PerCP-Cy5.5, or PE-Cy7. Antibody staining was performed following the manufacturer's protocol. Fluorescence-minus-one (FMO) controls were used to determine the gate of each antibody. Flow cytometry analysis was conducted using a FACS Aria flow cytometer (BD Bioscience, CA, USA), and data were analyzed by FlowJo software (Informer Technologies, USA).

\section{Sorting of microglia and macrophages}

Microglia of the mouse brain tissue were sorted by magnetic cell sorting (MACS) in combination with fluorescence-activated cell sorting (FACS). Single-cell suspensions of the brain tissue were prepared as described above ("Flow cytometry" section). Cells were stained with APC-conjugated anti-mouse CD45 antibody and PE-conjugated anti-mouse $\mathrm{CD} 11 \mathrm{~b}$ for $30 \mathrm{~min}$ at $4{ }^{\circ} \mathrm{C}$. Unstained antibody was washed in PBS and cells were incubated with anti-PE microbeads at $4{ }^{\circ} \mathrm{C}$ for 15 min. HBSS was used to wash off unlabeled microbeads. Cells labeled with primary antibody conjugated to $\mathrm{PE}$ were enriched by using MACS columns (Miltenyi Biotech, Bergisch Gladbach Germany) according to the explanatory memorandum, and then targeted microglia were gathered using the FACS Aria cell sorting system. Resident microglia were identified as the $\mathrm{CD} 45^{\text {int }} \mathrm{CD} 11 \mathrm{~b}^{+}$population, whereas infiltrated macrophages in the CNS were identified as the $\mathrm{CD} 11 \mathrm{~b}^{+} \mathrm{CD} 45^{\text {high }} \mathrm{F} 4 / 80^{+}$population. In addition, cell sorting of macrophages from the spleen was performed following the method in the "Flow cytometry" section to obtain the cell suspensions of the spleen. Then, cells were stained with APC-conjugated anti-mouse CD45 antibody, PE-conjugated anti-mouse CD11b, and FITCconjugated anti-mouse $\mathrm{F} 4 / 8030 \mathrm{~min}$ at $4{ }^{\circ} \mathrm{C}$. Unstained antibody was washed in PBS, and then targeted macrophages defined as the $\mathrm{CD} 45^{\text {high }} \mathrm{CD} 11 \mathrm{~b}^{+} \mathrm{F} 4 / 80^{+}$population were gathered using the FACS Aria cell sorting system. 
Isolated cells were collected in trizol reagent, vortexed, and kept at $-80^{\circ} \mathrm{C}$ for further experiments.

\section{Isolation of primary microglia}

Primary rat microglia culture was isolated as previously reported [35]. In brief, the cerebral cortices separated from neonatally 1-day-old rats and meninges were removed. Trypsinization was used to digest the striped cortical tissues for $30 \mathrm{~min}$, and $70-\mu \mathrm{m}$ nylon mesh cell strainer was used to obtain the mixed cortical cells. Cells were maintained in DMEM/F12 with fetal bovine serum (FBS), penicillin, and streptomycin (Gibco, Grand Island, NY, USA). Culture media were changed every 3 days until achieving a confluent monolayer at approximately 15 days. For the isolation of primary microglia, mild trypsinization was added to isolate microglia from the mixed glial cells. Purified microglia were cultured at $37^{\circ} \mathrm{C}$ under atmosphere condition for further experiments.

\section{Oxygen-glucose deprivation (OGD)}

To establish an ischemic-like condition in vitro, primary microglia were subjected to OGD as previously reported [36]. Briefly, microglia were cultured with serum-glucosedeprived cultures and placed in a hypoxic chamber with $95 \%$ nitrogen and $5 \% \mathrm{CO}_{2}$ for $5 \mathrm{~min}$ and sealed tightly. Subsequently, the chamber moved to an incubator under $5 \% \mathrm{CO}_{2} / 37^{\circ} \mathrm{C}$ for $3 \mathrm{~h}$. After the OGD treatment, serum and glucose-free medium were exchanged by glucosecontaining medium with or without rhFGF21, which was followed by incubating with $95 \%$ air and $5 \% \mathrm{CO}_{2}$ for $5 \mathrm{~h}$ and then analysis by qRT-PCR.

\section{Cell culture and treatment}

Primary cultured microglia and the BV2 cell line were used to characterize the effect of rhFGF21 on microglial polarization, inflammation cytokine release, and NF- $\mathrm{KB}$ and PPAR- $\gamma$ signaling activation. Cells were exposed to lipopolysaccharide (LPS) to induce polarized microglia and inflammatory secretion [37]. Briefly, microglia were treated with LPS $(250 \mathrm{ng} / \mathrm{mL})$ in the presence and absence of rhFGF21 $(100 \mathrm{nM})$ or PD173074 $(10 \mu \mathrm{M})$ for 4 h. Gene assays (involving IL-1 $\beta$, iNOS, TNF- $\alpha$, IL-6, CD86, CD206, Arg-1, IGF-1, and IL-10) were then detected in LPS-stimulated or OGD-treated primary microglia by qRT-PCR, and the effects of rhFGF21 on transcriptional activity of NF- $\mathrm{KB}$ in primary microglia were detected using immunofluorescence. Additionally, the polarization of microglia was analyzed by assessing the expression of the M1 marker CD86, which was identified by FACS staining.

\section{Western blot}

Total proteins of LPS-treated BV2 cells were purified by RIPA lysis supplemented with a protease and phosphatase inhibitor mixture. Protein concentrations were measured with a Bradford Protein Detection Kit. Then, $60 \mu \mathrm{g}$ of proteins from the samples and positive controls were separated on sodium dodecyl sulfate (SDS) polyacrylamide gels by electrophoresis. Subsequently, proteins were transferred onto PVDF membranes followed by blocking with primary antibodies FGFR1 (1:1000), p-FGFR1 (1:1000), NF- $\mathrm{B}$ (1:1000), PPAR- $\gamma(1: 400)$, and $\beta$-Actin (1:500) overnight at $4{ }^{\circ} \mathrm{C}$. Then, the membranes were incubated with secondary antibody donkey anti-rabbit IgG or goat antimouse IgG at a 1:10,000 dilution for $1 \mathrm{~h}$ at room temperature. Finally, the protein bands were detected with Image Lab software using Gel Doc Imager (Bio-Rad, Hercules, CA, USA) and the expression of target proteins was normalized against $\beta$-Actin.

\section{Immunofluorescence analysis}

Immunofluorescence staining was performed on paraffin brain sections as previously described [38]. Briefly, nonspecific binding of antibodies was blocked with 5\% BAS for $1 \mathrm{~h}$ at $37^{\circ} \mathrm{C}$ and the sections were then incubated with one or more primary antibodies against CD16/32, CD206, Iba1, or NF- $\mathrm{kB}$ in a dilution following the manufacturer's instruction at $4{ }^{\circ} \mathrm{C}$ overnight. After washing, secondary antibodies conjugated with adequate fluorochrome were added to visualize the expression of corresponding proteins and DAPI was used to stain the nuclei. Images of the penumbra of the infarct cortex were captured using a confocal laser scanning microscope (Laika, Japan). Data were analyzed with ImageJ (NIH Image, Bethesda, MD, USA) to calculate the fluorescence intensity or counting number of recognized cells per field.

\section{Statistical analysis}

All statistical analyses of the data were processed with Prism 7.0 software (GraphPad, San Diego, CA, USA) in a blinded manner. Data from individual groups were expressed as mean \pm SEM and characterized by a one-way ANOVA for multiple comparisons or Student's $t$ test (and nonparametric tests). Behavioral data were statistically analyzed by a two-way ANOVA for multiple comparisons. Statistical significance was considered at $P<0.05$ level.

\section{Results}

rhFGF21 protects against brain injury in MCAO mice

Previous reports have shown that rhFGF21 significantly reduces infarct volumes at $24 \mathrm{~h}$ after focal ischemia in rats compared with the vehicle treatment [39]. To assess the effect of rhFGF21 on brain injury after ischemic stroke in mice, infarct volumes were detected at 3 and 14 days after MCAO. The decreased infarct volume reached significance at 3 days after MCAO based on 2,3, 5-triphenyltetrazolium chloride (TTC) staining, and rhFGF21 also significantly reduced the infarct size at 
14 days after MCAO (Fig. 1a). The percentage of infarct volume in the MCAO group gradually decreased from $17.16 \pm 1.277$ at 3 days after MCAO to $9.657 \pm$ 1.189 at 14 days after $\mathrm{MCAO}$, and this percentage in the MCAO+rhFGF21 group decreased from $7.971 \pm$ 1.077 to $3.744 \pm 0.913$ (Fig. 1b). Moreover, behavioral assessments were evaluated at $1,3,7$, and 14 days after MCAO. Neurological deficits and feeling function in the rhFGF21 group, assessed by the modified neurological severity score (mNSS) test (Fig. 1c) and removal test (Fig. 1d, e), respectively, were significantly better than those in the MCAO group. Similarly, the rhFGF21-treated group exhibited a significant improvement in sensorimotor function, demonstrated by fewer right turns in the corner-turning test (Fig. 1f), and enhanced motor coordination, indicated by an increased latency to fall off the rotarod (Fig. 1g), compared to the vehicle-treated group at 14 days after MCAO. Together, these behavioral data suggest that post-stroke treatment with rhFGF21 facilitates functional recovery after MCAO.

rhFGF21 inhibits the inflammatory response in the cortex of the ischemic brain

The secretion of inflammatory cytokines plays a unique role in the inflammatory cascade reaction and neuronal injury following stroke. In this study, an array of inflammatory cytokines including IL- $1 \beta$, TNF- $\alpha$, IL-6, cyclooxygenase (COX)-2, monocyte chemoattractant protein (MCP)-1, and chemokine (C-X-C motif) ligand 1 (CXCL1) at $6,24,48$, and $72 \mathrm{~h}$ after stroke were analyzed by qRTPCR. mRNA expression of pro-inflammatory cytokines, including IL-6, TNF- $\alpha$, and CXCL1, were quickly increased after stroke and peaked at $24 \mathrm{~h}$ (Fig. 2b, d), whereas IL-1 $\beta$, COX-2, and MCP-1 levels peaked at $48 \mathrm{~h}$ (Fig. 2a, c, e) after stroke. However, rhFGF21 administration markedly suppressed the stroke-evoked enhancement of cytokine levels beginning at $24 \mathrm{~h}$, especially at 24 and $48 \mathrm{~h}$ after stroke (Fig. 2a-f). Interestingly, levels of IL-10, generally regarded as an anti-inflammatory cytokine, were robustly but transiently increased at $6 \mathrm{~h}$ after stroke; however, this high level of expression continued to $48 \mathrm{~h}$ in the rhFGF21 treatment groups (Fig. 2g). Furthermore, compared to vehicle, rhFGF21 significantly elevated the level of TGF- $\beta$ at $48 \mathrm{~h}$ (Fig. $2 \mathrm{~h}$ ). These results suggest that rhFGF21 ameliorates the MCAO-induced inflammatory response at the acute stage.

Additionally, IL-10, as an M2 marker, is commonly considered to be a mediator of microglial phenotype polarization [38], and TNF- $\alpha$, IL-1 $\beta$, IL-6, and MCP-1 are secreted by M1 microglia. Therefore, we speculate that rhFGF21 may affect microglia and their polarization.

\section{rhFGF21 modulates microglia polarization and the} temporal presence of microglial phenotypes and number in the ischemic brain

To investigate the potential impact of rhFGF21 on microglia in the brain after focal ischemic stroke, we first measured the number and phenotypes of resident

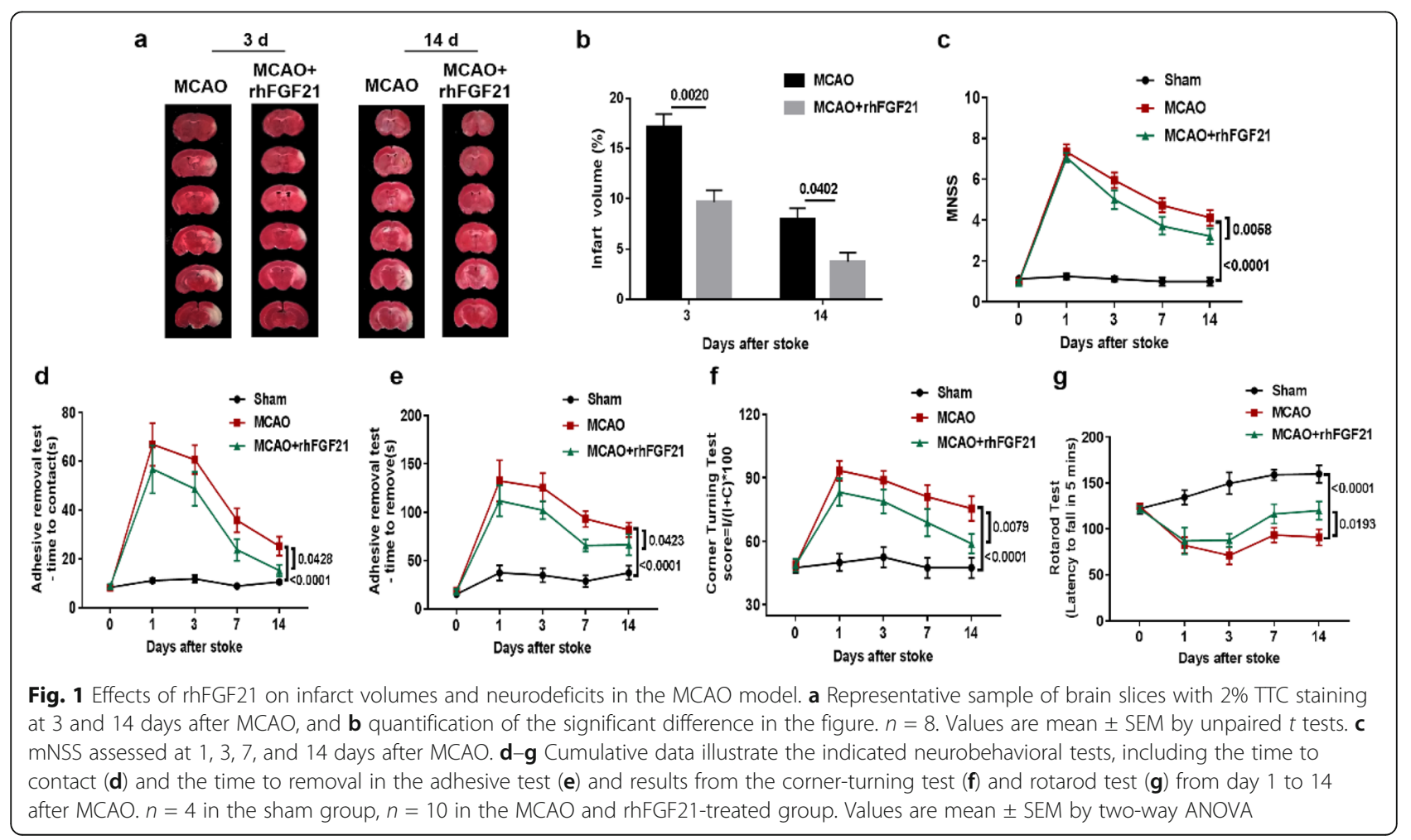



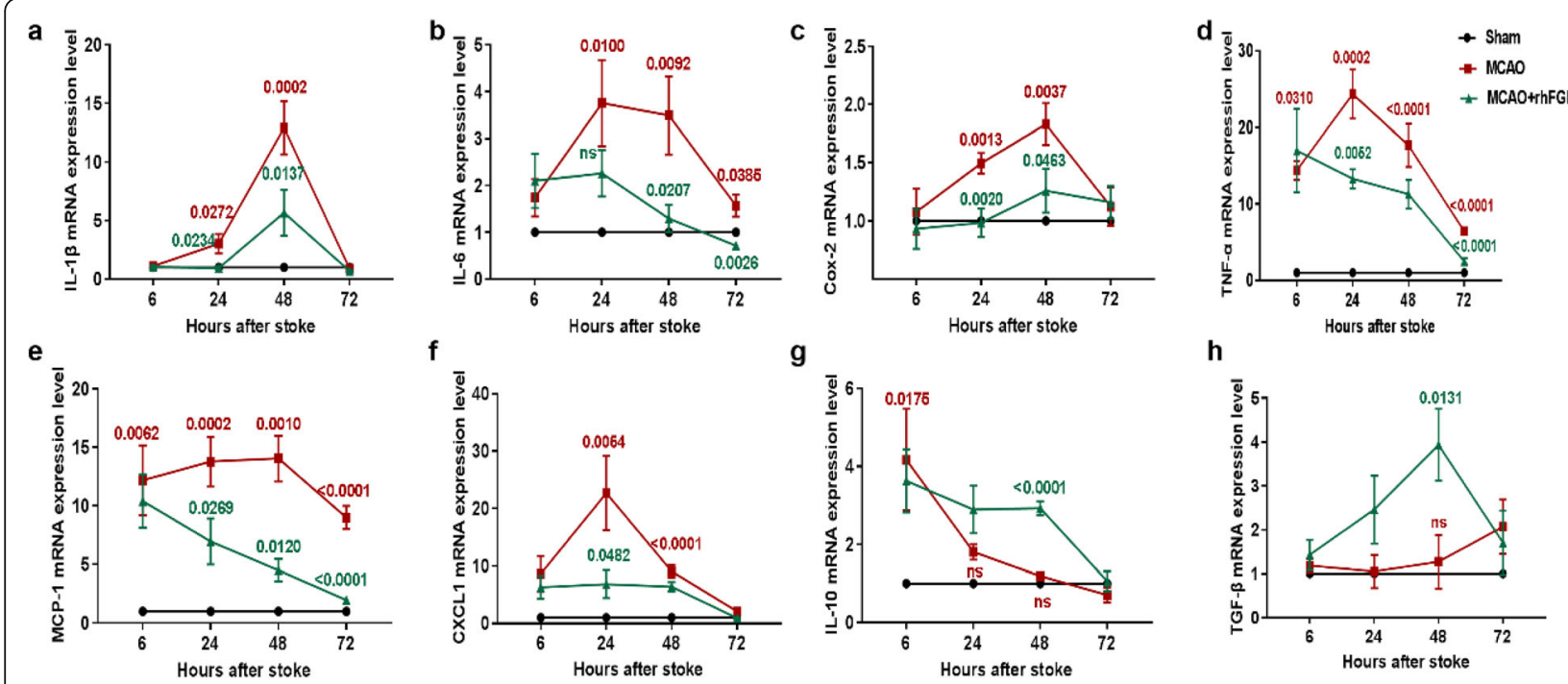

Fig. 2 Effects of rhFGF21 on inflammatory cytokines after MCAO. mRNA expression levels of IL-1 $\beta$ (a), IL-6 (b), COX-2 (c), TNF-a (d), MCP-1 (e), CXCL1 (f), IL-10 (g), and TGF- $\beta(\mathbf{h})$ in the cortex around the infarcted zone were detected via qRT-PCR at 6, 24, 48, and $72 \mathrm{~h}$ after MCAO. Values are mean \pm SEM by one-way ANOVA, $n=6$ per group. $p$ value (red) of MCAO versus sham, $p$ value (green) of MCAO versus MCAO+rhFGF21

microglia in the ischemic hemisphere by flow cytometry analysis at 3 days after stroke. Resident microglia were defined as $\mathrm{CD} 11 \mathrm{~b}^{+} \mathrm{CD} 45^{\text {int }}$ cells, and the populations of CD68, CD86, and CD206 microglia were gated using FMO controls (Fig. 3a). The MCAO group had significantly fewer microglia than the sham group, although there were no significant differences in the count of microglia between the vehicle-treated group and rhFGF21-treated group. Moreover, the counts of $\mathrm{CD} 68^{+}, \mathrm{CD} 86^{+}$, and $\mathrm{CD} 206^{+}$microglia in the ischemic hemisphere were significantly increased in the MCAO group compared with the sham group, and rhFGF21 obviously suppressed the expression of cell counts of the $\mathrm{CD} 8^{+}$and $\mathrm{CD} 86^{+}$microglia evoked by MCAO but did not markedly affect the variation of cell counts of CD206 ${ }^{+}$microglia (Fig. 3b).

The distribution of the microglial phenotype undergoes dynamic changes depending on timing and context; therefore, we further analyzed the variation in microglial phenotype at 1,3 , and 7 days after MCAO. The number of resident microglia was markedly lower than that in the sham group from 1 to 3 days after stroke; however, this returned to a similar level as that in the sham group by day 7 (Fig. 3c). Furthermore, the expression of CD68 was significantly upregulated beginning at 1 day following the ischemic event and continuing until 7 days poststroke. Importantly, rhFGF21 intervention significantly reversed the elevated CD68 expression, most strongly at 3 days and 7 days after stroke (Fig. 3d). CD86 has been classified as an M1 and M2b microglia marker that reduces the polarization of microglia to the M2a phenotype [37]. CD86 expression was significantly higher than that in the sham group at $24 \mathrm{~h}$ after MCAO but then decreased at day 3. However, the protein level of CD86 peaked at 7 days after $\mathrm{MCAO}$ at a level higher than that expressed at the other time points. Moreover, exposure to rhFGF21 treatment markedly suppressed the elevation of CD86 at 3 and 7 days after MCAO (Fig. 3e). Intriguingly, the number of $\mathrm{CD} 206^{+}$microglia transiently increased in the injured brain as early as 1 day after MCAO and then gradually decreased, consistent with a previous report [11]. However, compared with vehicle treatment, rhFGF21 did not significantly affect the expression of CD206 in microglia (Fig. 3f).

In addition, an immunohistochemistry approach was used to assess the expression of CD16/32, another marker of M1 microglia, in the penumbra of the infarct cortex and corresponding cortical tissue of animals at 3 days after MCAO (Fig. 3g). Consistently, the rhFGF21 treatment obviously attenuated the high expression of CD16/32 (Fig. 3h, i). Collectively, these findings suggest that post-stroke treatment with rhFGF21 inhibits the polarization of M1 microglia but does not facilitate the polarization of microglia toward the M2 phenotype.

\section{rhFGF21 reduces immune cell infiltration in the CNS and M1 macrophage accumulation}

Subsequently, we analyzed the accumulation of infiltrating immune cells defined as CD45 $5^{\text {high }}$ cells and the infiltration of $\mathrm{NK}\left(\mathrm{CD} 45^{\text {high }} \mathrm{CD} 3^{-} \mathrm{NK} 1.1^{+}\right), \mathrm{CD} 4^{+} \mathrm{T}$ $\left(\mathrm{CD} 45^{\text {high }} \mathrm{CD}^{+} \mathrm{CD}^{+}\right), \quad \mathrm{CD} 8^{+} \mathrm{T} \quad\left(\mathrm{CD} 45^{\text {high }} \mathrm{CD}^{+} \mathrm{CD}^{+}\right)$, neutrophils $\left(\mathrm{CD} 11 \mathrm{~b}^{+} \mathrm{CD} 45^{\text {high }} \mathrm{Ly}-6 \mathrm{G}^{+}\right)$, macrophages $\left(\mathrm{CD} 11 \mathrm{~b}^{+} \mathrm{CD} 45^{\text {high }} \mathrm{F} 4 / 80^{+}\right)$, and monocyte cells $\left(\mathrm{CD} 11 \mathrm{~b}^{+}\right.$ CD $45^{\text {high }}$ Ly- $6 \mathrm{C}^{+}$) in the CNS at 3 days after MCAO (Fig. 4a). Flow cytometry analysis revealed that rhFGF21 


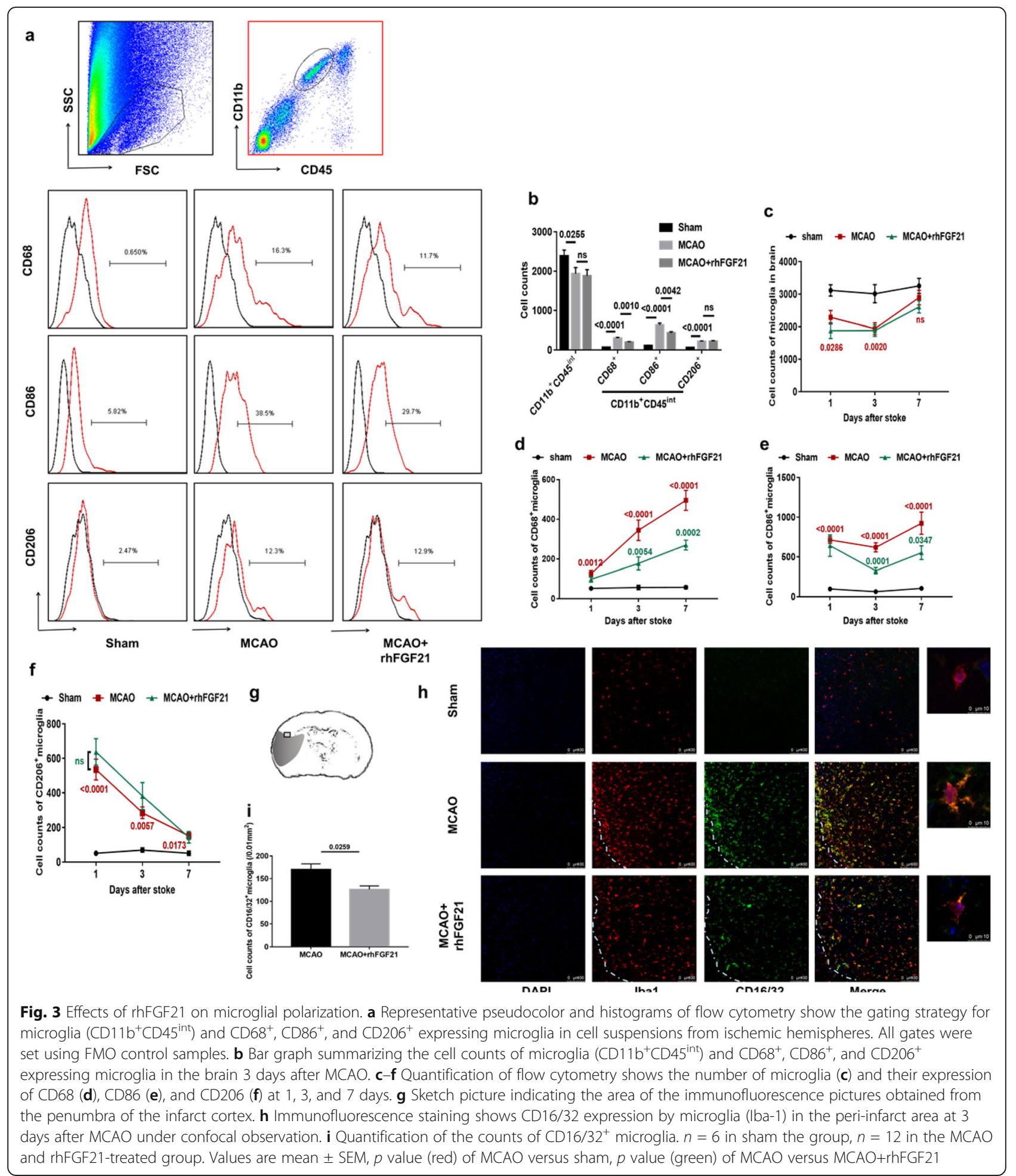

administration did not significantly affect the numbers of $\mathrm{NK}, \mathrm{CD} 4^{+} \mathrm{T}$, and $\mathrm{CD}^{+} \mathrm{T}$ cells that infiltrated in the CNS. However, rhFGF21 treatment of MCAO animals strikingly reduced the number of infiltrating immune cells defined as $\mathrm{CD} 45^{\text {high }}$ cells and macrophages $\left(\mathrm{CD} 11 \mathrm{~b}^{+} \mathrm{CD} 45^{\text {high }} \mathrm{F} 4 / 80^{+}\right)$compared with the vehicle treatment (Fig. 4c). Meanwhile, the number of neutrophils $\left(\mathrm{CD} 11 \mathrm{~b}^{+} \mathrm{CD} 45^{\text {high }}\right.$ Ly- $\left.6 \mathrm{G}^{+}\right)$and monocyte cells 


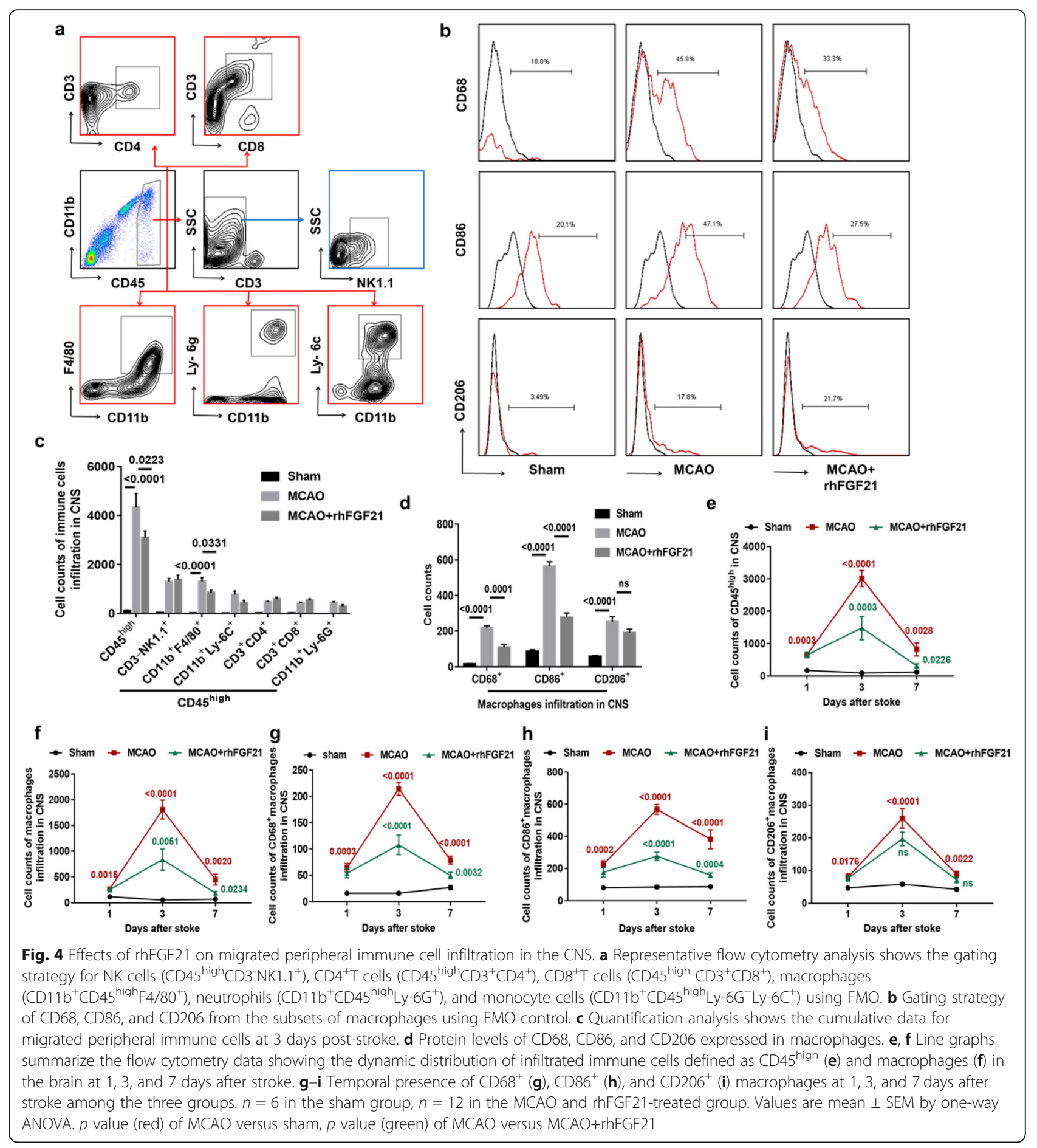

$\left(\mathrm{CD} 11 \mathrm{~b}^{+} \mathrm{CD} 45^{\text {high }} \mathrm{Ly}-6 \mathrm{C}^{+}\right)$in the rhFGF21-treated MCAO group was slightly lower than that in the vehicle-treated MCAO group.

In addition, the phenotype of infiltrating macrophages gated on $\mathrm{CD} 11 \mathrm{~b}^{+} \mathrm{CD} 45^{\text {high }} \mathrm{F} 4 / 80^{+}$was further detected by flow cytometry analysis at 3 days following stroke (Fig. 4b). The counts of CD68 ${ }^{+}$and $\mathrm{CD} 86^{+}$macrophages were significantly lower in rhFGF21-treated MCAO mice than in the vehicle-treated MCAO mice, although there were no significant differences in the count of $\mathrm{CD}_{206}{ }^{+}$macrophages between the vehicle-treated and rhFGF21-treated groups (Fig. 4d).

Moreover, we further detected the temporal profiles when infiltrating immune cells defined as CD $45^{\text {high }}$ cells, macrophages, and $\mathrm{CD}^{+} 8^{+}, \mathrm{CD} 6^{+}$, and $\mathrm{CD} 206^{+}$macrophages were present in the ischemic brain at 1,3 , 
and 7 days following stroke. The absolute counts of infiltrated immune cells (Fig. 4e), macrophages (Fig. 4f), and $\mathrm{CD}^{+} 8^{+}, \mathrm{CD}^{2} 6^{+}$, and $\mathrm{CD} 206^{+}$macrophages (Fig. $4 \mathrm{~g}-\mathrm{i}$ ) were dramatically increased in the CNS of MCAO mice, and they peaked at 3 days post-injury and subsequently returned to baseline levels by 7 days. Similar temporal profiles were also observed in rhFGF21-treated MCAO mice, but the cell counts of infiltrated immune cells (Fig. 4e), macrophages (Fig. 4f), and $\mathrm{CD}^{+} 8^{+}$and $\mathrm{CD} 86^{+}$ macrophages (Fig. 4g, h) that accumulated in CNS were significantly decreased in rhFGF21-treated MCAO mice compared with the vehicle-treated MCAO mice at 3 days and 7 days after stroke. Together, these results together suggest that rhFGF21 alleviates the accumulation of infiltrated immune cells (particularly macrophages) in the ischemic brain and might contribute to the inhibition of the macrophage-mediated inflammatory response.

\section{rhFGF21 suppresses the phenotypic alteration of macrophages toward the M1 in the spleen and blood}

Excessive stroke-induced immune responses lead to disturbances in peripheral immunity, which in turn interfere with immune cell infiltration in the injured brain [13]. rhFGF21 has been reported to inhibit macrophagemediated inflammation by suppressing NF- $\mathrm{BB}$ in RAW 264.7 cells [29]. To determine whether rhFGF21 alleviates CNS inflammation mediated by macrophagemediated peripheral inflammation, we performed a flow cytometry analysis to detect alterations in peripheral immune cell subsets in the spleen and blood at 3 days post-stroke. The gating strategies of neutrophils $\left(\mathrm{CD} 11 \mathrm{~b}^{+} \mathrm{Ly}_{6 \mathrm{G}^{+}}\right)$, monocytes $\left(\mathrm{CD} 11 \mathrm{~b}^{+} \mathrm{Ly} 6 \mathrm{C}^{+}\right), \mathrm{CD}^{+} \mathrm{T}$ cells, $\mathrm{CD}^{+}$cells, $\mathrm{NK} 1.1^{+}$cells, and macrophages $\left(\mathrm{CD} 11 \mathrm{~b}^{+} \mathrm{F} 4 / 80^{+}\right)$in single-cell suspensions from the spleen (Fig. 5a) and blood (Fig. 6a) were set by FMO control, and the gate settings of $\mathrm{CD}^{+} 8^{+}, \mathrm{CD} 86^{+}$, and $\mathrm{CD}^{2} 26^{+}$macrophages were set by FMO control in the spleen (Fig. 5c) and blood (Fig. 6c). After MCAO, the number of macrophages in the peripheral spleen organs was substantially diminished (Fig. 5b), although there was no significant difference in the blood (Fig. 6b). Notably, variation in other cell subsets was not observed in either the spleen or the blood. In contrast, the depletion of macrophages induced by MCAO was effectively alleviated by rhFGF21. Moreover, rhFGF21 significantly rescued the increased expression of CD68 and CD86 in macrophage cells residing in the spleen (Fig. 5d) and blood (Fig. 6d) following MCAO. However, there was no considerable difference in the expression of CD206 in the macrophages between the vehicle- and rhFGF21treated groups. These results suggest that rhFGF21 reduces macrophage activation in peripheral tissue, which is associated with the inflammatory processes in the CNS.
rhFGF2 1 regulates the secretion of IL-1 $\beta$, TNF- $\alpha$, IL- 6 , and TGF- $\beta$ cytokines in sorted microglia and macrophages at 3 days after stroke

To further detect the potential effects of rhFGF21 on the release of inflammatory cytokines in microglia and macrophages, we sorted microglia and infiltrated macrophages from the damaged hemisphere, and macrophages from the spleen to a purity above $90 \%$ and assessed gene expression by real-time PCR (Fig. 7a). Compared with the levels in the sham group, the expression levels of IL$1 \beta$ (5.33-fold) and TNF- $\alpha$ (1.42-fold) in microglia in the MCAO group were dramatically increased, but those of IL-6 (0.09-fold) and TGF- $\beta$ (0.28-fold) were significantly reduced. However, both the enhancement in IL-1 $\beta$ and TNF- $\alpha$ expression and the decline in IL-6 expression were hindered by the administration of rhFGF21, but TGF- $\beta$ expression was not significantly affected (Fig. $7 \mathrm{~b}$ ). In addition, upon induction of MCAO, the mRNA levels of IL-1 $\beta$ (11.70-fold), TNF- $\alpha$ (16.36-fold), and IL-6 (2.87-fold) in infiltrated macrophages were dramatically higher than those in macrophages from the spleen. In the presence of rhFGF21, IL-1 $\beta$ expression was remarkably inhibited, but TNF- $\alpha$ and IL- 6 expression levels were not significantly affected. In contrast, there were no differences in TGF- $\beta$ expression among all groups (Fig. 7c). Moreover, administration of rhFGF21 effectively attenuated the MCAO-induced increase in the levels of IL-1 $\beta$ (1.40-fold) and TNF- $\alpha$ (0.93-fold) in macrophages from the spleen but did not remarkably affect the levels of IL-6 (2.44-fold) or TGF- $\beta$ (1.08-fold) following MCAO (Fig. 7d). In summary, these outcomes further indicate that rhFGF21 mediates anti-inflammatory effects by modulating microglia and macrophages.

\section{rhFGF21 reduces M1 marker expression and pro- inflammatory cytokine secretion in primary microglia treated with oxygen-glucose deprivation (OGD) or lipopolysaccharide (LPS)}

To evaluate the effects of rhFGF21 on the polarization of microglia and pro-inflammatory cytokine production, we examined primary microglia stimulated by OGD or LPS. Similar results were observed, the administration of rhFGF21 markedly suppressed the expression of M1type genes (IL-1 $\beta$, iNOS, TNF- $\alpha$, IL-6, and CD86), but did not affect the M2-type genes (CD206, Arg-1, IGF-1, and IL-10) in OGD-treated primary microglia (Fig. 8a). Similarly, rhFGF21 markedly inhibited the mRNA level of iNOS and TNF- $\alpha$ in LPS-stimulated primary microglia (Fig. 8b), and the protein expression of CD86 was detected at the protein level using flow cytometry assays (Fig. 8c, d). Moreover, microglial activation during ischemic injury along with the activation of NF- $\mathrm{KB}$ is associated with the secretion of inflammatory cytokines. As shown in Fig. 8e, immunofluorescent staining $(\times 400)$ 


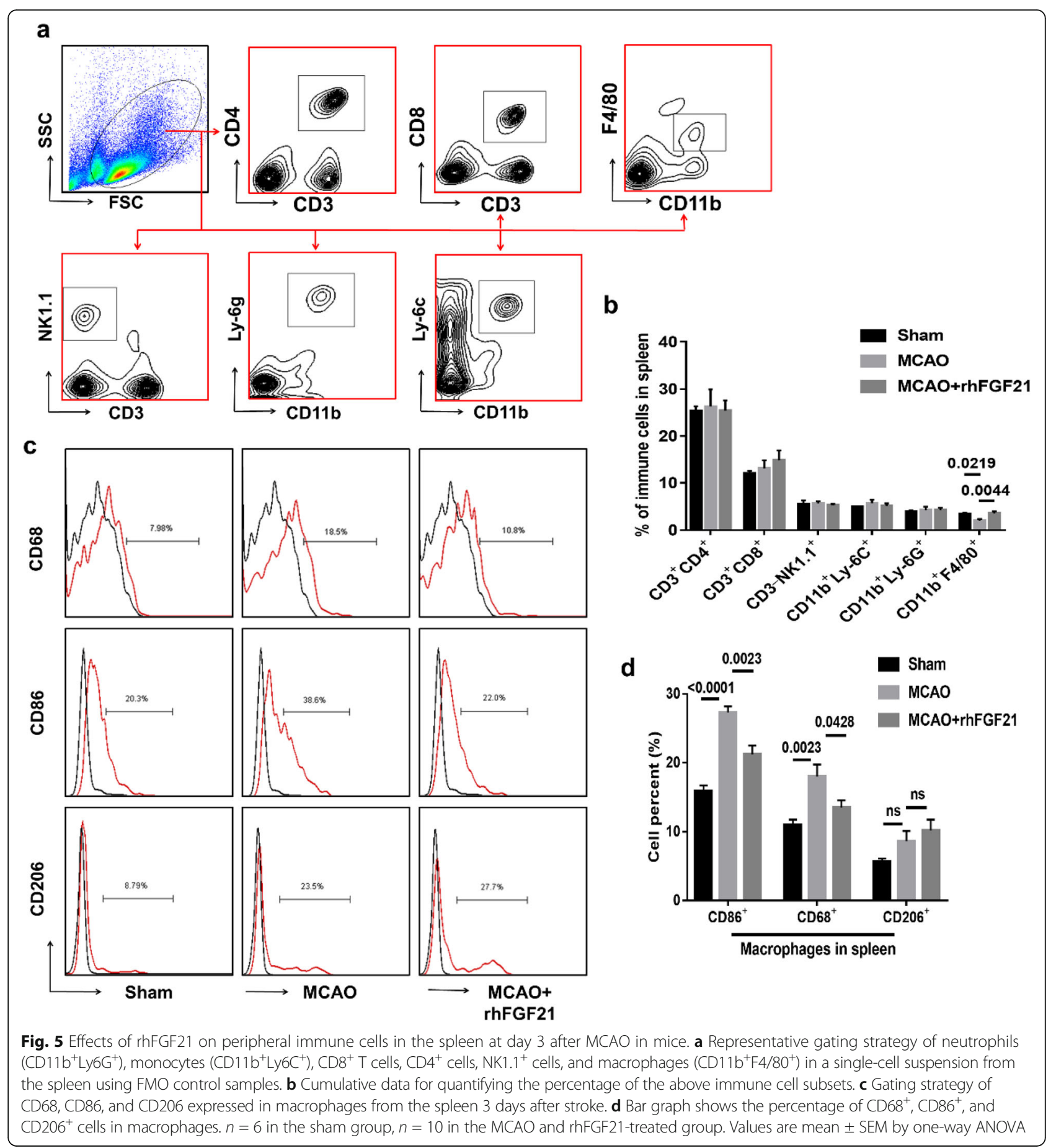

demonstrated that rhFGF21 suppressed the nuclear translocation of NF- $\mathrm{kB}$, indicating that the transphosphorylation activity of NF- $\mathrm{kB}$ was inhibited by rhFGF21. However, co-administration with PD173074, a selective inhibiter of FGFR1, reversed this effect of rhFGF21. Moreover, the counts of microglia for which $N F-k B$ is translocated into the nuclei are quantified in Fig. 8f, which indicates that the transcriptional activity of NF-kB was suppressed by rhFGF21. These data together demonstrate that rhFGF21 ameliorates microglia-mediated neuroinflammation by inhibiting $\mathrm{NF}-\mathrm{kB}$ signaling via the FGFR1 receptor.

rhFGF21 upregulates PPAR- $\gamma$ and inhibits the activation of NF-KB via FGFR1 in LPS-stimulated BV2 cells

NF- $\mathrm{kB}$ activation in microglia is associated with proinflammation, whereas the activity of PPAR- $\gamma$ is positively related to anti-inflammation. To evaluate whether 


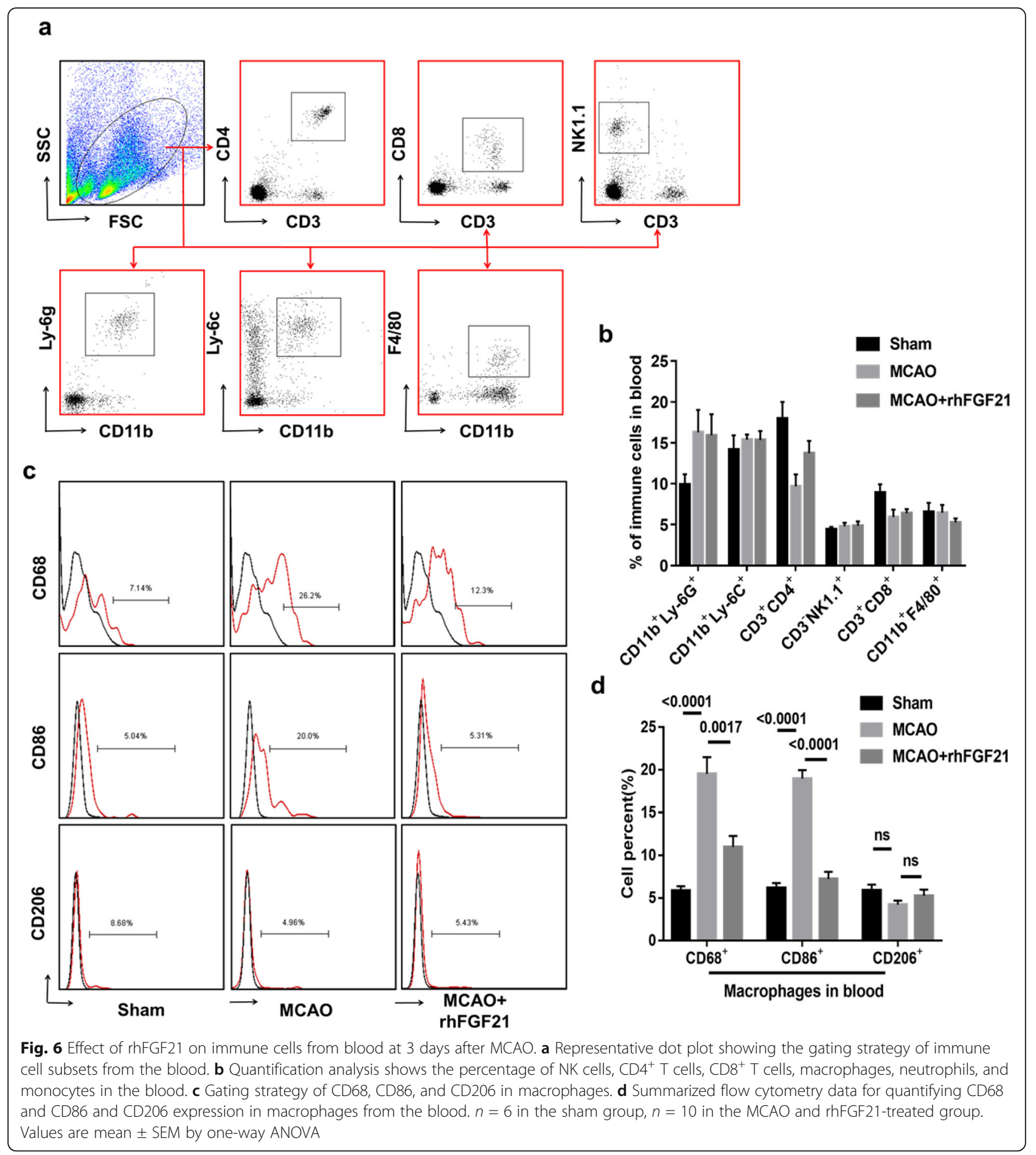

rhFGF21 activated PPAR- $\gamma$ and inhibited NF- $k B$, we performed western blot experiments in BV2 cells exposed to LPS. As expected, rhFGF21 significantly upregulated the phosphorylation level of FGFR1; however, PD173074 obviously reserved this upregulation (Fig. 9a, c). In addition, similar to observations in primary microglia, rhFGF21 markedly suppressed the transcriptional activity of NF-kB, which was reversed by PD173070 (Fig. 9b, d). Notably, rhFGF21 administration enhanced the expression of PPAR- $\gamma$ (Fig. 9b, e), which commonly alters M2 gene expression. Additionally, the effect of rhFGF21 was reversed by PD173074. These data indicated that rhFGF21 modulates microglial polarization via the NF- $k B$ and PPAR- $\gamma$ pathways. 


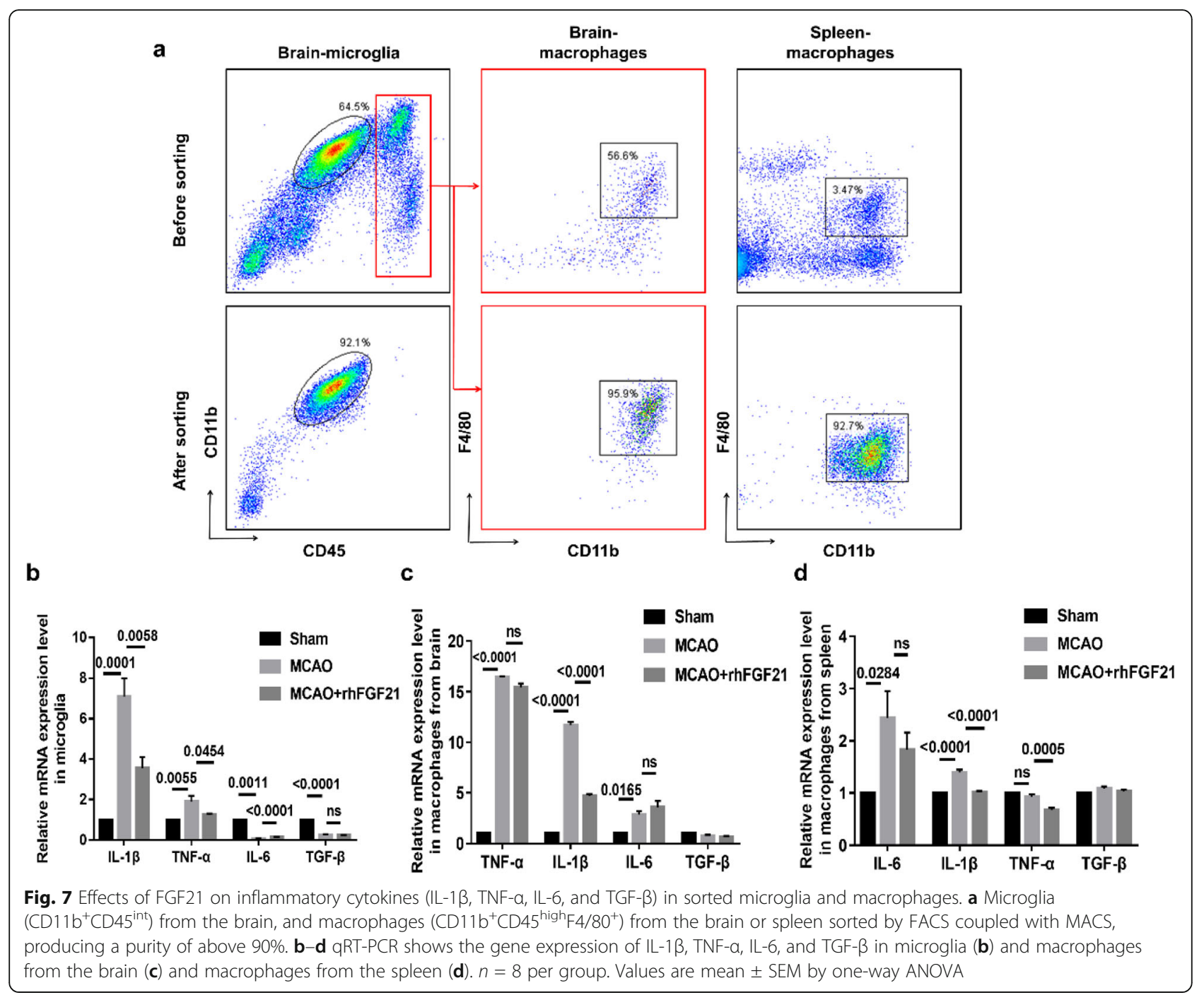

\section{Discussion}

The inflammatory response evoked by focal ischemia stroke is a complex and pleiotropic process [13]. The complexity is emphasized by the immunomodulation progression associated with multiple immune cells-resident microglia and an influx of hematogenous cells, that changes based on the time, space, and stage-specific milieu. The heterogeneity is highlighted by detrimental and protective immune effects mediated by those immune cells. Regulation of neuroinflammation has been recognized as an attractive approach for promising therapies in stroke. rhFGF21 is a safe and effective endocrine regulator that has been demonstrated to have strong anti-inflammatory effects, and it represents a promising candidate for microglia/macrophage-based therapy in acute stroke.

In the current study, we demonstrated the neuroprotective effects of rhFGF21 and highlighted its immunomodulatory effects by regulating resident microglia and hematogenous macrophages in acute ischemic stroke. Although this study is not the first to show that rhFGF21 may protect against cerebral ischemic injury in rats [39], it confirmed that rhFGF21 significantly reduced the infarct size and ameliorated the neurological deficit in mice affected by stroke through a set of experiments (including TTC and behavior assessment). Moreover, our study also revealed that rhFGF21 remarkably dampened the upregulation of pro-inflammatory gene expression. Therefore, our study provides evidence that these outcomes associated with the neuroprotective effects of rhFGF21 are tightly linked with its antiinflammatory function.

Post-ischemic inflammation is a hallmark of ischemic stroke pathology, which plays critical roles in acute brain damage and profoundly affects long-term recovery [40, 41]. Inflammation-associated conditions regulated by pro-inflammatory and anti-inflammatory cytokines are associated with impaired neurogenesis and neuronal 


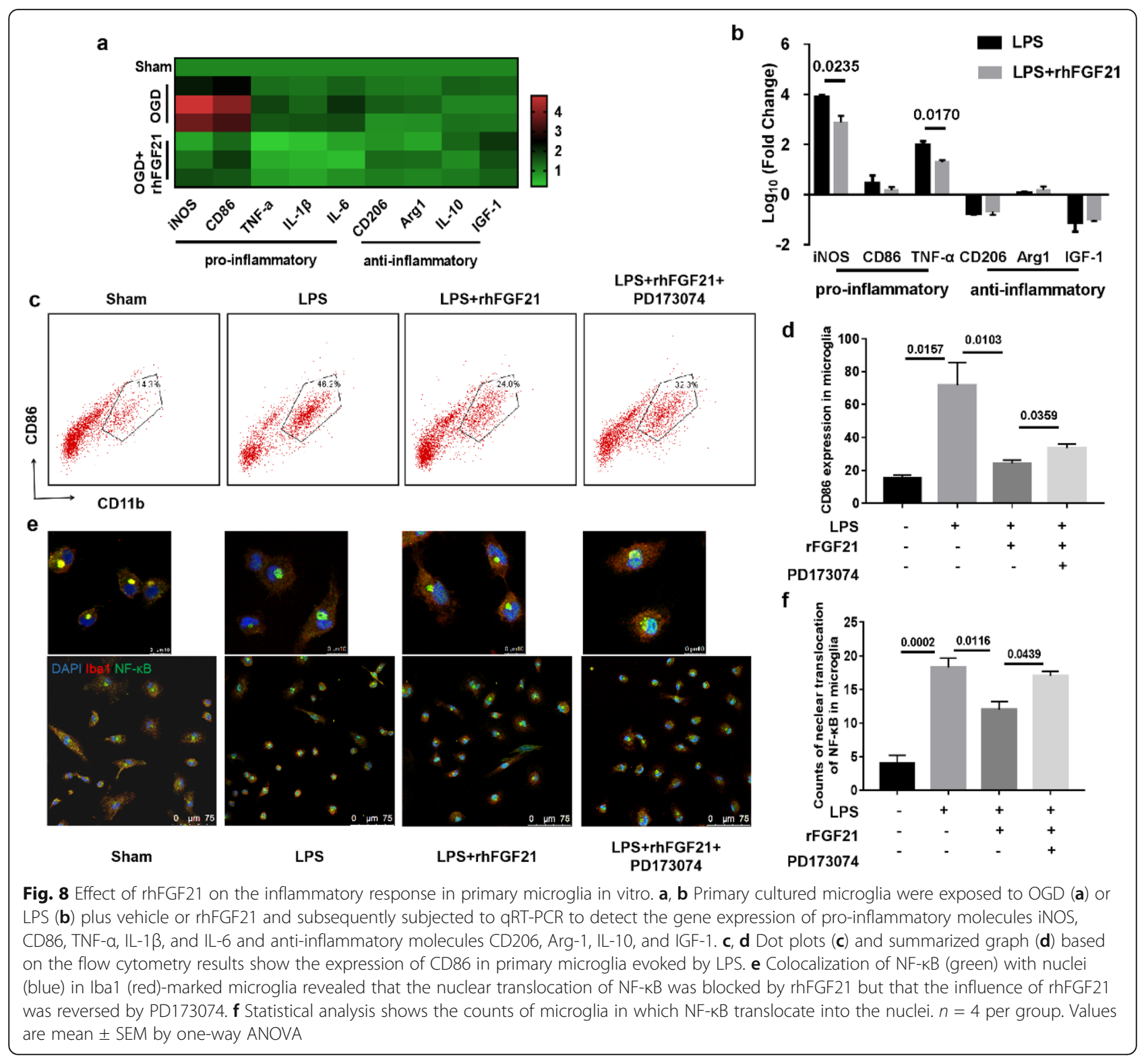

survival [42]. Our findings are consistent with a previous report [28] that showed that almost all inflammatory cytokines were upregulated immediately following stroke, with levels peaking at 24 or $48 \mathrm{~h}$ after stroke. However, the increase in IL-10 expression appears strongly but transiently as early as $6 \mathrm{~h}$ post-stroke. Among the cytokines detected, TNF- $\alpha$ has both neurotoxic and neuroprotective effects, while IL-1 $\beta$ have characteristically neurotoxic effects. Both TNF- $\alpha$ and IL-1 $\beta$ are mainly produced by microglia and macrophages [43] and synthesized by segregated subsets [44]. The cellular source of IL-6 remains controversial, although it is likely predominantly expressed in threatened neurons and activated microglia around the infarct region and targeted at neurons or microglia, and it contributes to both the damage and repair processes [45]. TGF- $\beta$, as an antiinflammatory cytokine, is associated with tissue repair. We next investigated the effect of rhFGF21 on inflammatory gene (TNF- $\alpha$, IL-1 $\beta$, IL-6, TGF- $\beta$ ) expression in microglia and invading peripheral macrophages, which are defined as the major cellular contributors to neuroinflammation [46, 47]. Although there are analogous phenotypic and functional characteristics among these inflammatory genes, numerous studies have revealed that they may play unique roles under pathological conditions [48]. Zarruk et al. [49] detected higher expression of TNF- $\alpha$ in microglia than in macrophages of LysMEGFP knock-in mice and higher expression of IL-1 $\beta$ and Arg-1 in macrophages than in macroglia in a permanent MCAO model. In our model, rhFGF21 significantly 


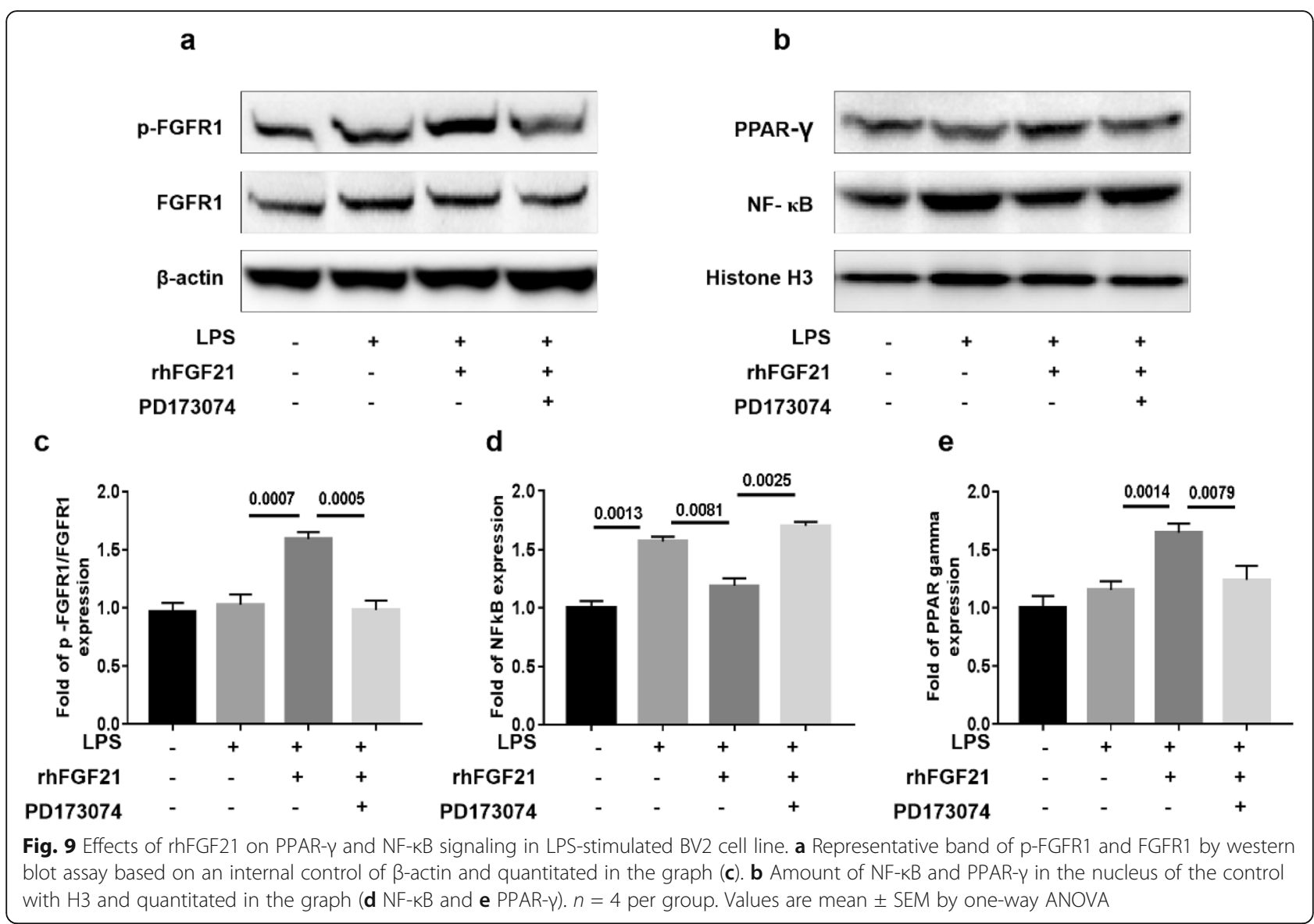

reduced the level of IL-1 $\beta$ expression not only in microglia and infiltrated macrophages but also in splenic macrophages. Surprisingly, the mRNA level of IL-6 in resident microglia isolated from MCAO mice was far lower than that in sham mice. Although we have no suitable explanation for this phenomenon, investigating the temporal pattern of the source of IL- 6 may provide a reasonable explanation.

Microglia are major cellular contributor to post-injury inflammation and have the potential to act as a key factor for disease onset and progression and contribute to the neurological outcome of acute brain injury [50]. Under pathological conditions, microglia are rapidly activated and undergo dramatic morphological and phenotypic changes accompanied by the induction of inflammatory cytokines. The classical activation phenotype (M1) is an inflammatory phenotype that produces pro-inflammatory cytokines, while the alternative activation phenotype (M2) is an anti-inflammatory phenotype that is characterized by the secretion of anti-inflammatory cytokines [51]. Our findings further demonstrated that rhFGF21 attenuated the polarization of microglia toward M1 but have no effect on the M2 phenotype in the acute phase of the MCAO model. Consistently, our in vitro results demonstrated that
rhFGF21 hampered the expression of pro-inflammatory cytokines in LPS- or OGD-treated microglia but had no influence on anti-inflammatory genes (IL-10, CD206, and IGF-1). Concomitantly, mice that underwent experimental MCAO exhibited a gradual decrease in the ischemic hemisphere from day 1 to 3 after reperfusion, and the level subsequently returned to preinjury levels by day 7 . A similar phenomenon was also described by a previous study [52] in which microglia from the ischemic hemisphere were remarkably reduced at 3 days after stroke. Furthermore, our results revealed the temporal profile of microglial polarization. In accordance with previous research [53], we observed that the levels of the M1-type marker (CD68) significantly increased beginning from day 1 onward. Notably, the expression of the M2 marker CD206 was also increased at 1 day after MCAO, although this increase was no longer observed within 7 days of MCAO injury, which is consistent with the findings of Perego et al. [54]. Taking into consideration the earlier upregulation of IL-10 gene expression, which mediates the shift of microglia to the M2 phenotype, there may be a temporary increase in M2-like microglia at 1 to 3 days post-stroke, and this notion is supported by the results of Kanazawa et al. [4]. 
Moreover, our study focused on the temporal and spatial presence of migrated immune cells in the acute phase of stroke and highlighted the participation of peripheral macrophages. A previous study [55] showed that the different immune cell types in the post-ischemic area had distinct temporal profiles while the majority of immune cells dramatically accumulated in the ischemic hemisphere at 3 days after stroke. Similarly, in our findings, a massive accumulation of immune cells occurred at 3 days after reperfusion, and the levels were restored back to baseline by day 7 . Importantly, rhFGF21 effectively eliminated the invasion of peripheral immune cells. Additionally, rhFGF21 suppressed the activation of peripheral macrophages in the spleen and blood of mice subjected to MCAO, which is consistent with previous reports showing that FGF21 reduced macrophage-mediated inflammation by NF-kB in RWA264.7 macrophages [29]. These findings suggest that the anti-inflammatory effects of rhFGF21 are mediated not only by resident microglia in the brain but also by hematogenous macrophages.

$\mathrm{NF}-\mathrm{KB}$ is a key transcription factor in the progression of inflammation, and its activation is accompanied by the release of a panel of inflammatory cytokines and chemokines, such as TNF- $\alpha$, IL-1 $\beta$, IL- 6 , and Cox- $2[56$, 57]. Indeed, microglia polarization has been proposed to induce multiple mechanisms, including NF- $\mathrm{kB}$ signaling pathways [58]. Previous literature demonstrated that the beneficial effects of rhFGF21 on macrophages occur through the inhibition of NF- $k B$, and our study further validated that rhFGF21 suppresses the activity of NF- $\mathrm{KB}$ via FGFR1 in LPS-stimulated murine microglia. In addition, PPAR- $\gamma$ is a nuclear transcriptional factor [59], and its activation affects not only peripheral systems in ischemia-reperfusion-induced kidney injury and trinitrobenzenesulfonic acid (TNBS)-induced inflammatory bowel disease but also the CNS due to its antiinflammatory ability [28]. In the present study, rhFGF21 significantly elevated the transcriptional activities of PPAR- $\gamma$ in LPS-stimulated BV2 cells, which further contributed to anti-inflammation. To recapitulate, rhFGF21, through its actions on FGFR1, suppresses the inflammatory response by modulating the activation of microglia via inhibiting NF- $\mathrm{kB}$ and elevating PPAR- $\gamma$.

\section{Conclusions}

In summary, our studies demonstrate that the antiinflammatory effect of rhFGF21 on focal cerebral ischemia occurs through regulation of both central microglia/macrophages and peripheral macrophages via $N F-\kappa B$ and PPAR- $\gamma$ signaling pathways, indicating that rhFGF21 is a promising candidate for the treatment of ischemic stroke.

\section{Abbreviations}

MCAO: Transient middle cerebral artery occlusion; BBB: Blood-brain barrier; MACS: Magnetic cell sorting; CCA: Common carotid artery; ECA: External carotid artery; ICA: Internal carotid artery; FACS: Fluorescence-activated cell sorting; tPA: Tissue plasminogen activator; TNF-a: Tumor necrosis factor-a; iNOS: Inducible nitric oxide synthase; IL-1 $\beta$ : Interleukin-1 $\beta$; IL-6: Interleukin-6; TGF- $\beta$ : Transforming growth factor beta; IGF-1: Insulin-like growth factor 1 ; IL10: Interleukin-1; IL-4: Interleukin-4; MCP-1: Monocyte chemoattractant protein-1; CXCL1: Chemokine C-X-C motif ligand 1; HBSS: Hank's balanced salt solution; PBS: Phosphate-buffered saline; ANOVA: One-way analysis of variance; CNS: Central nervous system; rhFGF21: Recombinant human fibroblast growth factor 21; NF-kB: Nuclear factor-kappa B; PPAR-

$\gamma$ : Peroxisome proliferator-activated receptor- $\gamma$

\section{Acknowledgements}

We thank the staffs at the Laboratory of Pharmaceutical Sciences of Wenzhou Medical University and Neurosurgery of First Affiliated Hospital of Wenzhou Medical University for providing experimental space, facilities, and technical services.

\section{Authors' contributions}

DW performed the experiments, analyzed the results, and wrote the manuscript. FL did the tMCAO surgery, and flow analysis was performed by PL. LZ and FH were major contributors in cell culture. YX, LL, XT, and XW conceived of the study, commented on the results, and revised the manuscript. The final manuscript was approved by all authors.

\section{Funding}

This project was supported by the National Natural Science Foundation of China (No. 81771284, 81971180), Scientific Research Project of Wenzhou (No. ZY2019001), General Scientific Research Project of Zhejiang Provincial Department of Education (No. Y201942264), and Research Unit of Research and Clinical Translation of Cell Growth Factors and Diseases, Chinese Academy of Medical Science (No. 2019RU010).

\section{Availability of data and materials}

The datasets used and/or analyzed during the current study are available from the corresponding author on reasonable request.

\section{Ethics approval and consent to participate}

All surgical procedures and experimental protocols were approved by the Animal Care and Use Committee of Wenzhou Medical University in Wenzhou (IACUC no: 2018-242)

Consent for publication

Not applicable.

\section{Competing interests}

The authors declare that they have no competing interests.

\section{Author details}

${ }^{1}$ Department of Neurosurgery, First Affiliated Hospital of Wenzhou Medical University, Wenzhou 325035, Zhejiang, China. ${ }^{2}$ School of Pharmaceutical Sciences, Wenzhou Medical University, Wenzhou 325035, Zhejiang, China

Received: 15 February 2020 Accepted: 10 August 2020

Published online: 31 August 2020

References

1. Anttila JE, et al. Role of microglia in ischemic focal stroke and recovery: focus on Toll-like receptors. Prog Neuro-Psychopharmacol Biol Psychiatry. 2017;79(Pt A):3-14

2. Writing Group, M, et al. Heart Disease and Stroke Statistics-2016 update: a report from the American Heart Association. Circulation. 2016;133(4):e38-360.

3. Dirnagl U. Pathobiology of injury after stroke: the neurovascular unit and beyond. Ann N Y Acad Sci. 2012;1268:21-5.

4. Kanazawa M, et al. Microglia and monocytes/macrophages polarization reveal novel therapeutic mechanism against stroke. Int J Mol Sci. 2017; 18(10):2135.

5. Lee GA, et al. Interleukin 15 blockade protects the brain from cerebral ischemia-reperfusion injury. Brain Behav Immun. 2018;73:562-70.

6. Spangenberg EE, Green KN. Inflammation in Alzheimer's disease: lessons learned from microglia-depletion models. Brain Behav Immun. 2017;61:1-11. 
7. Jones KA, et al. Peripheral immune cells infiltrate into sites of secondary neurodegeneration after ischemic stroke. Brain Behav Immun. 2018;67:299-307.

8. Ziko l, et al. Neonatal overfeeding alters hypothalamic microglial profiles and central responses to immune challenge long-term. Brain Behav Immun. 2014:41:32-43.

9. Moskowitz MA, Lo EH, ladecola C. The science of stroke: mechanisms in search of treatments. Neuron. 2010;67(2):181-98.

10. Ransohoff RM, Cardona AE. The myeloid cells of the central nervous system parenchyma. Nature. 2010;468(7321):253-62.

11. Ma Y, et al. The biphasic function of microglia in ischemic stroke. Prog Neurobiol. 2017;157:247-72.

12. Tang $Y$, Le W. Differential roles of $M 1$ and $M 2$ microglia in neurodegenerative diseases. Mol Neurobiol. 2016;53(2):1181-94.

13. Kim E, Cho S. Microglia and monocyte-derived macrophages in stroke. Neurotherapeutics. 2016:13(4):702-18.

14. Ryden M. Fibroblast growth factor 21: an overview from a clinical perspective. Cell Mol Life Sci. 2009;66(13):2067-73.

15. Hsuchou H, Pan W, Kastin AJ. The fasting polypeptide FGF21 can enter brain from blood. Peptides. 2007;28(12):2382-6.

16. Tan BK, et al. Fibroblast growth factor 21 (FGF21) in human cerebrospinal fluid: relationship with plasma FGF21 and body adiposity. Diabetes. 2011; 60(11):2758-62.

17. Lin Z, et al. Fibroblast growth factor 21 prevents atherosclerosis by suppression of hepatic sterol regulatory element-binding protein-2 and induction of adiponectin in mice. Circulation. 2015;131(21):1861-71.

18. Yan X, et al. FGF21 deletion exacerbates diabetic cardiomyopathy by aggravating cardiac lipid accumulation. J Cell Mol Med. 2015;19(7):1557-68.

19. Yu Y, et al. Fibroblast growth factor 21 protects mouse brain against Dgalactose induced aging via suppression of oxidative stress response and advanced glycation end products formation. Pharmacol Biochem Behav. 2015;133:122-31.

20. Huang $X$, et al. The cell adhesion molecule $L 1$ regulates the expression of FGF21 and enhances neurite outgrowth. Brain Res. 2013;1530:13-21.

21. Li SM, et al. Treatment of CIA mice with FGF21 down-regulates TH17-IL-17 axis. Inflammation. 2016;39(1):309-19.

22. Hui $X$, et al. The FGF21-adiponectin axis in controlling energy and vascular homeostasis. J Mol Cell Biol. 2016:8(2):110-9.

23. Gomez-Samano MA, et al. Fibroblast growth factor 21 and its nove association with oxidative stress. Redox Biol. 2017;11:335-41.

24. Tanajak P, Chattipakorn SC, Chattipakorn N. Effects of fibroblast growth factor 21 on the heart. J Endocrinol. 2015;227(2):R13-30.

25. Kurosu $\mathrm{H}$, et al. Tissue-specific expression of betaKlotho and fibroblast growth factor (FGF) receptor isoforms determines metabolic activity of FGF19 and FGF21. J Biol Chem. 2007;282(37):26687-95

26. Bookout $A L$, et al. FGF21 regulates metabolism and circadian behavior by acting on the nervous system. Nat Med. 2013;19(9):1147-52

27. Chen J, et al. FGF21 Protects the blood-brain barrier by upregulating PPARgamma via FGFR1/beta-klotho after traumatic brain injury. J Neurotrauma. 2018;35(17):2091-103.

28. Pan J, et al. Malibatol A regulates microglia M1/M2 polarization in experimental stroke in a PPARgamma-dependent manner. J Neuroinflammation. 2015;12:51.

29. Yu Y, et al. Fibroblast growth factor 21 (FGF21) inhibits macrophagemediated inflammation by activating Nrf2 and suppressing the NF-kappaB signaling pathway. Int Immunopharmacol. 2016:38:144-52.

30. Jiang $Y$, et al. Endocrine regulator rFGF21 (recombinant human fibroblast growth factor 21) improves neurological outcomes following focal ischemic stroke of type 2 diabetes mellitus male mice. Stroke. 2018:49(12):3039-49.

31. Wang $\mathrm{H}$, et al. High-level expression and purification of soluble recombinant FGF21 protein by SUMO fusion in Escherichia coli. BMC Biotechnol. 2010;10:14

32. Jin WN, et al. Depletion of microglia exacerbates postischemic inflammation and brain injury. J Cereb Blood Flow Metab. 2017;37(6):2224-36.

33. Feng $Y$, et al. Infiltration and persistence of lymphocytes during late-stage cerebral ischemia in middle cerebral artery occlusion and photothrombotic stroke models. J Neuroinflammation. 2017;14(1):248.

34. Bao $Y$, et al. A role for spleen monocytes in post-ischemic brain inflammation and injury. J Neuroinflammation. 2010;7:92.

35. Lin $L$, et al. Characteristics of primary rat microglia isolated from mixed cultures using two different methods. J Neuroinflammation. 2017;14(1):101.
36. Li M, et al. Astrocyte-derived interleukin-15 exacerbates ischemic brain injury via propagation of cellular immunity. Proc Natl Acad Sci U S A. 2017; 114(3):E396-405.

37. Chhor $\mathrm{V}$, et al. Characterization of phenotype markers and neuronotoxic potential of polarised primary microglia in vitro. Brain Behav Immun. 2013; 32:70-85.

38. Jin Q, et al. Improvement of functional recovery by chronic metformin treatment is associated with enhanced alternative activation of microglia/ macrophages and increased angiogenesis and neurogenesis following experimental stroke. Brain Behav Immun. 2014;40:131-42.

39. Yang $X$, et al. Design and evaluation of lyophilized fibroblast growth factor 21 and its protection against ischemia cerebral injury. Bioconjug Chem. 2018:29(2):287-95.

40. Li S, et al. Early histone deacetylase inhibition mitigates ischemia/ reperfusion brain injury by reducing microglia activation and modulating their phenotype. Front Neurol. 2019;10:893.

41. Xie W, et al. HMGB1-triggered inflammation inhibition of notoginseng leaf triterpenes against cerebral ischemia and reperfusion injury via MAPK and NF-kappaB signaling pathways. Biomolecules. 2019;9(10):512.

42. Loppi S, et al. HX600, a synthetic agonist for RXR-Nurr1 heterodimer complex, prevents ischemia-induced neuronal damage. Brain Behav Immun 2018;73:670-81.

43. Lambertsen $\mathrm{KL}$, Biber K, Finsen B. Inflammatory cytokines in experimental and human stroke. J Cereb Blood Flow Metab. 2012:32(9):1677-98.

44. Clausen $\mathrm{BH}$, et al. Interleukin-1 beta and tumor necrosis factor-alpha are expressed by different subsets of microglia and macrophages after ischemic stroke in mice. J Neuroinflammation. 2008;5:46.

45. Suzuki S, Tanaka K, Suzuki N. Ambivalent aspects of interleukin-6 in cerebral ischemia: inflammatory versus neurotrophic aspects. J Cereb Blood Flow Metab. 2009;29(3):464-79.

46. Zheng ZV, et al. The Dynamics of Microglial Polarization Reveal the Resident Neuroinflammatory Responses After Subarachnoid Hemorrhage. Transl Stroke Res, 2020:11(3):433-49.

47. Kronenberg G, et al. Distinguishing features of microglia- and monocytederived macrophages after stroke. Acta Neuropathol. 2018;135(4):551-68.

48. Chu HX, et al. Immune cell infiltration in malignant middle cerebral artery infarction: comparison with transient cerebral ischemia. J Cereb Blood Flow Metab. 2014;34(3):450-9.

49. Zarruk JG, Greenhalgh AD, David S. Microglia and macrophages differ in their inflammatory profile after permanent brain ischemia. Exp Neurol. 2018; 301(Pt B):120-32.

50. Poh $L$, et al. Evidence that NLRC4 inflammasome mediates apoptotic and pyroptotic microglial death following ischemic stroke. Brain Behav Immun. 2019;75:34-47.

51. Yang $X$, et al. Resveratrol regulates microglia M1/M2 polarization via PGC1alpha in conditions of neuroinflammatory injury. Brain Behav Immun. 2017; 64:162-72.

52. Ritzel RM, et al. Functional differences between microglia and monocytes after ischemic stroke. J Neuroinflammation. 2015;12:106.

53. Hu X, et al. Microglia/macrophage polarization dynamics reveal novel mechanism of injury expansion after focal cerebral ischemia. Stroke. 2012; 43(11):3063-70

54. Perego C, Fumagalli S, De Simoni MG. Temporal pattern of expression and colocalization of microglia/macrophage phenotype markers following brain ischemic injury in mice. J Neuroinflammation. 2011;8:174

55. Gelderblom M, et al. Temporal and spatial dynamics of cerebral immune cell accumulation in stroke. Stroke. 2009:40(5):1849-57.

56. Zusso M, et al. Ciprofloxacin and levofloxacin attenuate microglia inflammatory response via TLR4/NF-kB pathway. J Neuroinflammation. 2019:16(1):148.

57. Li Y, et al. Galectin-1 attenuates neurodegeneration in Parkinson's disease model by modulating microglial MAPK/lkappaB/NFkappaB axis through its carbohydrate-recognition domain. Brain Behav Immun. 2020;83:214-25.

58. He $\mathrm{Y}$, et al. Thiamet $\mathrm{G}$ mediates neuroprotection in experimental stroke by modulating microglia/macrophage polarization and inhibiting NF-kappaB p65 signaling. J Cereb Blood Flow Metab. 2017;37(8):2938-51.

59. Odegaard JI, et al. Macrophage-specific PPARgamma controls alternative activation and improves insulin resistance. Nature. 2007;447(7148):1116-20.

\section{Publisher's Note}

Springer Nature remains neutral with regard to jurisdictional claims in published maps and institutional affiliations. 